\title{
Correction to: A comparison of the observed and expected prevalence of HIV in persons released from Ontario provincial prisons in 2010
}

\author{
Ryan Van Meer ${ }^{1} \cdot$ Tony Antoniou ${ }^{2,3,4} \cdot$ Daniel McCormack $^{2} \cdot$ Sumeet Khanna ${ }^{3}$ - Claire Kendall ${ }^{2,5,6} \cdot$ Lori Kiefer $^{7,8}$. \\ Fiona G. Kouyoumdjian ${ }^{2,9,10}$
}

Published online: 31 July 2019

(C) The Canadian Public Health Association 2019

\section{Correction to: Canadian Journal of Public Health https://doi.org/10.17269/s41997-019-00233-0}

The Acknowledgements section was inadvertently omitted from this article; it appears in its entirety below.

Acknowledgements This study was funded by the Ontario HIV Treatment Network. This study was also supported by ICES, which is funded by an annual grant from the Ontario Ministry of Health and LongTerm Care (MOHLTC). We acknowledge the Ontario Ministry of Community Safety and Correctional Services (MCSCS), which provided data for the study, and we appreciate the contributions of Mr. Michael Kirk to the study. The analyses, opinions, results, and conclusions reported in this paper are those of the authors, and no endorsement by the Ontario HIV Treatment Network, ICES, the MOHLTC, or the MCSCS is intended or should be inferred.
Parts of this material are based on data and/or information compiled and provided by the Canadian Institute for Health Information (CIHI). However, the analyses, conclusions, opinions, and statements expressed in the material are those of the authors, and not necessarily those of CIHI.

TA is supported by a New Investigator Award from the Canadian Institutes of Health Research (CIHR) and a Clinician Investigator Award from the University of Toronto Department of Family and Community Medicine. CK is supported by a New Investigator Award from CIHR.

Publisher's note Springer Nature remains neutral with regard to jurisdictional claims in published maps and institutional affiliations.
The online version of the original article can be found at https://doi.org/ 10.17269/s41997-019-00233-0

\section{Fiona G. Kouyoumdjian}

kouyouf@mcmaster.ca

1 Department of Health Research Methods, Evidence and Impact, McMaster University, Hamilton, Ontario, Canada

2 ICES, Toronto, Ontario, Canada

3 Department of Family and Community Medicine, St. Michael's Hospital and University of Toronto, Toronto, Canada

4 Li Ka Shing Knowledge Institute, St. Michael's Hospital, Toronto, Canada

5 C.T. Lamont Primary Health Care Research Group, Bruyère Research Institute, Ottawa, Canada
Department of Family Medicine, University of Ottawa, Ottawa, Canada

7 Dalla Lana School of Public Health, University of Toronto, Toronto, Ontario, Canada

8 Ministry of Community Safety and Correctional Services, Toronto, Ontario, Canada

9 Department of Family Medicine, McMaster University, 100 Main Street West, Hamilton, Ontario L8P 1H6, Canada

10 MAP Centre for Urban Health Solutions, St. Michael's Hospital, Toronto, Ontario, Canada 